\title{
Fixed Point Results for Multivalued Mappings with Applications
}

\author{
Arshad Khan, ${ }^{1}$ Muhammad Sarwar $\mathbb{D}^{1}{ }^{1}$ Farhan Khan, ${ }^{1}$ Habes Alsamir $\mathbb{D},{ }^{2}$ \\ and Hasanen A. Hammad ${ }^{3}$ \\ ${ }^{1}$ Department of Mathematics, University of Malakand, Chakdara Dir (L), Pakistan \\ ${ }^{2}$ College of Business Administration-Finance Department, Dar Al Uloom University, Saudi Arabia \\ ${ }^{3}$ Department of Mathematics, Faculty of Science, Sohag University, Sohag 82524, Egypt
}

Correspondence should be addressed to Habes Alsamir; habes@dau.edu.sa

Received 17 March 2021; Revised 8 July 2021; Accepted 7 August 2021; Published 24 August 2021

Academic Editor: Santosh Kumar

Copyright (C) 2021 Arshad Khan et al. This is an open access article distributed under the Creative Commons Attribution License, which permits unrestricted use, distribution, and reproduction in any medium, provided the original work is properly cited.

In this manuscript, using the concept of multivalued contractions, some new Banach- and Caristi-type fixed point results are established in the context of metric spaces. For the reliability of the presented results, some examples and applications to Volterra integral type inclusion are also studied. The established results unify and generalize some existing results from the literature.

\section{Introduction and Preliminaries}

Volterra integral equations appear in different scientific applications such as in the spread of epidemics, semiconductor devices, and population dynamics. Also, the dynamics of multispan uniform continuous beams subjected to a moving load is one of the best applications of Volterra integral equations. A universal method for finding sorption uptake curves of fluid multicomponent mixtures in porous solid at variable and constant concentration of mixture components on the basis of the Volterra integral equation has been proposed. The fixed point theory for multivalued mappings is a serious subject of set-valued analysis. In Banach spaces, several wellknown fixed point theorems of single-valued mappings such as Banach and Schauder have been extended to multivalued mappings. There are a lot of applications for multivalued mappings such as optimal control theory, differential inclusions, game theory, and many branches in physics.

The Banach contraction principle is used in a variety of fields of mathematics. This technique has many applications in studying the existence of solutions for nonlinear Volterra integral equations, and nonlinear integrodifferential equations in Banach spaces.

Recently, it has been widely spread. For example, in abstract spaces, the Fredholm integral equation introduced by Fredholm [1] and the solutions of Fredholm- and
Volterra-type integral equations have been discussed analytically by Rezan et al. [2-4], Rus [5], Aydi et al. [6], Karapinar et al. [7], and Hammad and De la Sen $[8,9]$ and numerically by Panda et al. $[10,11]$ and Berenguer et al. [12].

Throughout this paper, the symbols $N(M), C(M), C B($ $M)$, and $C P(M)$ refer to the family of all nonempty, nonempty closed, nonempty closed bounded, and nonempty compact subset of $M$, respectively.

For multivalued contraction mappings, we have the following.

Definition 1 (see [13]). Suppose that $(M, d)$ is a metric space. Denote by $C B(M)$, the set of all nonempty closed bounded subsets of $M$. The Pompeiu-Hausdorff metric $P_{H M}: C B(M$ )$\times C B(M) \longrightarrow[0, \infty)$ induced by the distance $d$ is formulated as follows: for all $A, B \in C B(M)$,

$$
P_{H M}(A, B)=\max \left\{\sup _{s \in B} d(s, A), \sup _{t \in A} d(t, B)\right\},
$$

where $d(s, A)=\inf _{t \in A} d(s, t)$. Also, the pair $\left(C B(M), P_{H M}\right)$ is called a generalized Hausdorff distance induced by $d$.

In 1969, the stipulation of Banach in single-valued mappings was modified to multivalued mappings by Nadler [13] as follows. 
Theorem 2. Let $(M, d)$ be a complete metric space (CMS) and $T$ be a multivalued mapping on $M$ so that $T(s)$ is a nonempty closed, bounded subset of $M$. If for each $s \in M$ there is $c \in(01)$ so that

$$
P_{H M}(T(s), T(t)) \leq c d(s, t), \quad \forall s, t \in M,
$$

then, $T$ has a fixed point in $M$.

In the literature, via abstract spaces, some authors obtained nice fixed point results for contractive mappings under certain conditions, for example, Hussain et al. [14] prove the existence of several fixed point results in ordinary and partially order metric spaces by studying the notion of Geraghty-type contractive mapping via simulation function along with $C$-class function. In integral type, Branciari [15] introduced some common fixed point results under general contractive conditions.

In Meir-Keeler type, Agarwal et al. [16] obtained some exciting fixed point results. In Menger probabilistic metric spaces, Chauhan et al. [17] discussed a hybrid coincidence and common fixed point theorem under a strict contractive condition with an application. Via the notion of $\alpha$-admissible mapping, the existence of fixed point theorems under $w$-distance mappings with an application is presented by Kutbi and Sintunavarat [18], and the others concerned with studying the notion of multivalued mapping and its contributions in fixed point theory such as Nadler [13], Ali and Kamran [19], Aubin and Siegel [20], Covitz and Nadler [21], Hot [22], and Ali et al. [23].

$\mathrm{Du}$ and Karapinar [24] introduce the concept of a Caristi-type cyclic map and present a new convergence theorem and a best proximity point theorem for Caristi-type cyclic maps. Petrusel and Sîntămarian [25] obtained a new result in the link of single-valued and multivalued Caristitype mappings. Hussain et al. [26] introduce the notion of Suzuki-type multivalued contraction with simulation functions and then set up some new fixed point and data dependence results for these types of contraction mappings. Karapinar [27] used lower semicontinuous mappings to generalize Caristi-Kirk's fixed point theorem on partial metric spaces. Abdeljawad and Karapinar [28] generalize CristiKirik's fixed point theorem to Cone metric spaces using Cone-valued lower semicontinuous maps. The relation between Caristi's result and its restriction to the function verifying Caristi's stipulations with continuous real functions is explained by Jachymski [29]. Khojasteh et al. [30] introduce the idea that many known fixed point theorems can easily be derived from the Caristi theorem. Also, Karapinar et al. [31] proposed a new fixed point theorem which is inspired from both Caristi and Banach.

In 2013, Ali et al. [32] initiated the idea of generalized $\alpha_{*}$ -admissible mappings. Via this concept, fixed point consequences to generalized Mizoguchi's fixed point theorem are derived. For single-valued mappings, Caristi [33] introduced an important theorem in fixed point field and called it the "Caristi fixed point theorem." This theorem was generalized to multivalued mappings in Banach spaces by Feng and Liu [34] as follows:
Theorem 3. Assume that $(M, d)$ is a CMS and $T: M \longrightarrow$ $N(M)$ is a multivalued mapping so that $T(s)$ is a closed subset of $M$, if there is a constant $c \in(0,1)$ so that

$$
d(t, T(t)) \leq c d(s, t)
$$

for all $s \in M$ and $t \in I_{b}^{s}$, where

$$
I_{b}^{s}=\{t \in T(s): b d(s, t) \leq d(s, T(s)), b \in(0,1)\},
$$

then, there is a fixed point of $T$ in $M$ with $c<b$ and $d(s, T(s)$ ) is lower semicontinuous.

Theorem 4. Assume that $(M, d)$ is a CMS and $T: M \longrightarrow$ $C(M)$ is a multivalued mapping so that $T(s)$ is a nonempty subset of $M$. Let $\phi: M \longrightarrow \mathbb{R}$ be a lower semicontinuous and bounded from below function and $p:[0, \infty) \longrightarrow 0, \infty)$ be a nondecreasing, continuous, and subadditive function so that $p^{-1}(\{0\})=\{0\}$. If for each $s \in X$, there is an element $t$ $\in T(s)$ verifying

$$
p(d(s, t)) \leq \phi(s)-\phi(t)
$$

then, the mapping $T$ has a fixed point on $M$.

Recently, Isik et al. [35] generalized Banach contraction and Caristi's fixed point theorem for a single-valued map as the following:

Theorem 5. Let $(M, d)$ be a CMS and $T: M \longrightarrow M$ be a selfcontinuous mapping, if there is the mapping $\varphi:[0, \infty] \longrightarrow[$ $0, \infty]$ so that $\lim _{s \longrightarrow 0^{+}} \varphi(s)=0, \varphi(0)=(0)$, and

$$
d(T s, T t) \leq \varphi(d(s, t))-\varphi(d(T s, T t)), \quad \forall s, t \in M
$$

then, there is a unique fixed point of $T$.

Theorem 6. Assume that $(M, d)$ be a CMS and $T: M \longrightarrow M$ is a self-mapping. Let $W$ be a set of mappings $f: \mathbb{R} \longrightarrow(0$, $\infty)$ so that the hypotheses in the following hold:

(1) $f$ is continuous and strictly increasing

(2) For each sequence $a_{n} \subseteq \mathbb{R}^{+}, \lim _{n \rightarrow \infty} a_{n}=0$ if and only if $\lim _{n \longrightarrow \infty} f\left(a_{n}\right)=1$

(3) For all $a, b \in \mathbb{R}, f(a+b) \leq f(a) f(b)$

If the function $\phi: M \longrightarrow R$ is lower semicontinuous and bounded from as follows so that

$$
f(d(s, T s)) \leq \frac{f(\phi(s))}{f(\phi(T s))}, \quad \forall s \in M
$$

then, T has a fixed point.

Definition 7 (see [36]). A function $p:[0, \infty) \longrightarrow 0, \infty)$ is called subadditive if $p(a+b) \leq p(a)+p(b)$ for every $a, b \in 0$ $, \infty)$. 
Definition 8 (see [13]). Suppose that $T: M \longrightarrow N(M)$ be a multivalued mapping, a point $s \in M$ is called a fixed point of $T$ if $s \in T(M)$.

Definition 9 (see [37]). Let $(M, d)$ be a metric space $A$ and $B$ be nonempty subsets of $M$. Then, a set-valued mapping $T$ $: A \cup B \longrightarrow A \cup B$ is called a set-valued cyclic map if $T(A)$ $\subset B$ and $T(B) \subset A$, where $T(A)=\cup\{T x: x \in A\}$.

The lemma in the following is very useful in the sequel.

Lemma 10 (Zorn's lemma). Let $S$ be a partially order set. If every totally ordered subset of $S$ has an upper bound then $S$ contains a maximal element.

Lemma 11 (see [10]). Assume that $A$ and $B$ are closed and bounded subset of $M$ and let $a \in A$. For each positive $\eta>$ 0 , then there is $b \in B$ so that $d(a, b) \leq P_{H M}(A, B)+\eta$. Moreover, if $B$ is a compact then there is $b \in B$ so that $d(a, b)$ $\leq P_{H M}(A, B)$.

The focus of this work is extending Theorems 3, 4, 5, and 6 for multivalued mappings via generalized contractive conditions. An example and application for the existence of solution of Volterra integral inclusion are also given.

\section{Fixed Point Results}

We begin this section with the first main result.

Theorem 12. Let $(M, d)$ be a complete metric space and $T$ $: M \longrightarrow C(M)$ be a multivalued mapping. If there is a nondecreasing mapping $\varphi:[0 \infty) \longrightarrow 0 \infty)$ so that $\lim _{t \rightarrow 0^{+}} \varphi(t)=0$, $\varphi(0)=0$ and for all $s \in M$, there is $t \in I_{b}^{s}$, so that

$$
d(t, T t) \leq \varphi(d(s, t))-\varphi\left(\frac{d(t, T t)}{b}\right),
$$

where $I_{b}^{s}=\{t \in T(s): b d(s, t) \leq d(s, T(s)), b \in(0,1)\}$ and $d(s$ , Ts) is lower semicontinuous; then, $T$ has a fixed point in $M$.

Proof. Let $s_{\circ}$ be an arbitrary element in $M$. Since $I_{b}^{s}$ is nonempty for any $b \in(0,1)$, we can build a sequence $\left\{s_{n}\right\}$ with $s_{n+1} \in I_{b}^{s_{n}}$ for each $n \in \mathbb{N} \cup\{0\}$ so that

$$
d\left(s_{n+1}, T s_{n+1}\right) \leq \varphi\left(d\left(s_{n}, s_{n+1}\right)\right)-\varphi\left(\frac{1}{b} d\left(s_{n+1}, T s_{n+1}\right)\right) .
$$

Since $s_{n+2} \in I_{b}^{s+1}$, then, we can write

$$
d\left(s_{n+1}, s_{n+2}\right) \leq \frac{1}{b} d\left(s_{n+1}, T s_{n+1}\right) .
$$
have

Because the mapping $\varphi$ is nondecreasing, so by (9), we

$$
0 \leq d\left(s_{n+1}, T s_{n+1}\right) \leq \varphi\left(d\left(s_{n}, s_{n+1}\right)\right)-\varphi\left(d\left(s_{n+1}, s_{n+2}\right)\right) .
$$

This implies that the sequence $\left\{\varphi\left(d\left(s_{n}, s_{n+1}\right)\right)\right\}$ is nonincreasing. Since $\varphi$ is bounded as follows, then there is $k \in \mathbb{R}$ so that $\varphi\left(d\left(s_{n}, s_{n+1}\right)\right) \longrightarrow k$ as $n \longrightarrow \infty$.

Now, for $m, n \in \mathbb{N}$ with $m>n$, we get

$$
\begin{aligned}
d\left(s_{n}, s_{m}\right) & \leq \sum_{i=n}^{m-1} d\left(s_{i}, s_{i+1}\right) \leq \frac{1}{b} \sum_{i=n}^{m-1} d\left(s_{i}, T s_{i}\right) \\
& \leq \frac{1}{b} \sum_{i=n}^{m-1}\left[\varphi\left(d\left(s_{i-1}, s_{i}\right)\right)-\varphi\left(d\left(s_{i}, s_{i+1}\right)\right)\right] \\
& \leq \frac{1}{b}\left(\varphi\left(d\left(s_{n-1}, s_{n}\right)\right)-\varphi\left(d\left(s_{m-1}, s_{m}\right)\right)\right) .
\end{aligned}
$$

Passing the limit in the above inequality as $n, m \longrightarrow \infty$, one can write

$$
\lim _{n, m \longrightarrow \infty} d\left(s_{n}, s_{m}\right)=0,
$$

this proves that $\left\{s_{n}\right\}$ is a Cauchy sequence. The completeness of $M$ leads to there is $s \in M$ so that $\lim _{n \rightarrow \infty} d\left(s_{n}, s\right)=0$. Since $d\left(s_{n}, T s_{n}\right)$ is lower semicontinuous, decreasing, $\varphi$ is nondecreasing and by (9), we obtain that

$$
\begin{aligned}
0 \leq d\left(s_{n+1}, T s_{n+1}\right) & =\varphi\left(d\left(s_{n}, s_{n+1}\right)\right)-\varphi\left(\frac{1}{b} d\left(s_{n+1}, T s_{n+1}\right)\right) \\
& =\varphi\left(\frac{1}{b} d\left(s_{n}, T s_{n}\right)\right)-\varphi\left(\frac{1}{b} d\left(s_{n+1}, T s_{n+1}\right)\right),
\end{aligned}
$$

or, equivalently,

$$
d\left(s_{n+1}, T s_{n+1}\right)<d\left(s_{n}, T s_{n}\right),
$$

this implies

$$
d\left(s_{n+1}, T s_{n+1}\right)<d\left(s_{n}, T s_{n}\right),
$$

therefore, $d\left(s_{n}, T s_{n}\right)$ converges to zero. Again, because $d\left(s_{n}\right.$ ,$\left.T s_{n}\right)$ is lower semicontinuous, then, we have

$$
0 \leq d(s, T s) \leq \lim _{n \longrightarrow \infty} d\left(s_{n}, T s_{n}\right)=0 .
$$

Hence, $d(s, T s)=0$; also, the closed property of $T(s)$ implies that $s \in T s$.

Remark 13. Theorem 12 is more general than Theorem 3, because if $T$ verifies the stipulation of Theorem 3, then for each $s \in M$, there is $t \in I_{b}^{s}$ so that

$$
d(t, T t) \leq c d(s, t) \leq \frac{c}{1+c / b-\sqrt{c / b}} d(s, t)
$$


this insinuate that

$$
\left(1+\frac{c}{b}-\sqrt{\frac{c}{b}}\right) d(t, T t) \leq c d(s, t)
$$

this equivalent to

$$
\left(1-\sqrt{\frac{c}{b}}\right) d(t, T t)+\left(\frac{c}{b}\right) d(t, T t) \leq c d(s, t),
$$

yields

$$
d(t, T t) \leq \frac{c d(s, t)}{1-\sqrt{c / b}}-\frac{c}{1-\sqrt{c / b}}\left(\frac{d(t, T t)}{b}\right)
$$

Setting $\varphi(p)=c p /(1-\sqrt{c / b})$, we have

$$
d(t, T t) \leq \varphi(d(s, t))-\varphi\left(\frac{d(t, T t)}{b}\right) .
$$

Moreover, $d(s, T s)$ is lower semicontinuous; therefore, $T$ has a fixed point by Theorem 12 .

If we put $\varphi(p)=p^{2}$ for all $p \geq 0$ in Theorem 12 , we get the result as follows.

Corollary 14. Suppose that $(M, d)$ is a CMS and $T: M$ $\longrightarrow C(M)$ is a multivalued mapping. If for any $s \in M$ there is $t \in I_{b}^{s}$ so that

$$
d(t, T t) \leq(d(s, t))^{2}-\left(\frac{d(t, T t)}{b}\right)^{2},
$$

then, there exists a fixed point of $T$.

Theorem 15. Let $(M, d)$ be a CMS and $T: M \longrightarrow C P(M)$ be a multivalued mapping, where $C P(M)$ is a compact subset of $M$. If there exists a nondecreasing mapping $\varphi:[0, \infty) \longrightarrow 0$, $\infty)$ so that $\lim _{t \rightarrow 0} \varphi(t)=0, \varphi(0)=0$, and

$$
P_{H M}(T s, T t) \leq \varphi(d(s, t))-\varphi\left(P_{H M}(T s, T t)\right), \quad \forall s, t \in M,
$$

then, there is a fixed point of $T$ in $M$.

Proof. Consider $s_{\circ}$ is an arbitrary point of $M$. According to Lemma 11 , construct a sequence $\left\{s_{n}\right\}$ with $s_{n+1} \in T s_{n}$ for all $n \in N$ so that

$$
\begin{aligned}
0 \leq d\left(s_{n}, s_{n+1}\right) & \leq P_{H M}\left(T s_{n-1}, T s_{n}\right) \\
& \leq \varphi\left(d\left(s_{n-1}, s_{n}\right)\right)-\varphi\left(P_{H M}\left(T s_{n-1}, T s_{n}\right)\right) \\
& \leq \varphi\left(d\left(s_{n-1}, s_{n}\right)\right)-\varphi\left(d\left(s_{n}, s_{n+1}\right)\right)
\end{aligned}
$$

this implies that $\left\{\varphi\left(d\left(s_{n}, s_{n+1}\right)\right)\right\}$ is a nonincreasing sequence, as $\varphi$ is bounded from the following so there is $r$ $\in \mathbb{R}$ so that $\lim _{n \rightarrow \infty} \varphi\left(d\left(s_{n}, s_{n+1}\right)\right)=r$.
To show that $\left\{s_{n}\right\}$ is a Cauchy sequence, let $m, n \in N$ with $m>n$ and applying Lemma 11 , we have

$$
\begin{aligned}
d\left(s_{n}, s_{m}\right) & \leq \sum_{i=n}^{m-1} d\left(s_{i}, s_{i+1}\right) \\
& \leq \sum_{i=n}^{m-1} P_{H M}\left(T s_{i-1}, T s_{i}\right) \\
& \leq \sum_{i=n}^{m-1}\left[\varphi\left(d\left(s_{i-1}, s_{i}\right)\right)-\varphi\left(d\left(s_{i}, s_{i+1}\right)\right)\right] \\
& =\left[\varphi\left(d\left(s_{n-1}, s_{n}\right)\right)-\varphi\left(d\left(s_{m-1}, s_{m}\right)\right)\right] .
\end{aligned}
$$

As $n, m \longrightarrow \infty$, we get

$$
\lim _{n, m \longrightarrow \infty} d\left(s_{n}, s_{m}\right)=0 \text {. }
$$

Thus, $\left\{s_{n}\right\}$ is a Cauchy sequence. The completeness of $M$ leads to there is $s_{\circ} \in M$ so that $\lim _{n \longrightarrow \infty} s_{n}=s_{\circ}$. Thus, the sequence $T s_{n}$ converges to $T s_{0}$. Since $s_{n+1} \in T s_{n}$ for each $n$ $\in N$, this implies that $s_{\circ} \in T s_{\circ}$. This finishes the proof.

Example 1. Let

$$
\begin{gathered}
M=\left\{s_{i}=\frac{i(i+1)}{2} i \in N\right\}, \\
d(s, t)=|s-t| .
\end{gathered}
$$

It is obvious that the pair $(M, d)$ is complete metric space. Define a multivalued mapping $T$ by

$$
T(s)= \begin{cases}s_{1}, & \text { if } s=s_{1} \wedge h \in M g \\ \left\{s_{1}, s_{2}, \cdots, s_{i-1}\right\}, & \text { if } s=s_{i} \text { and } i>1 .\end{cases}
$$

Now, we verify that the multivalued mapping $T$ satisfied the condition of Theorem 15 with $\varphi(p)=p \cdot \exp (p)$. Note that $P_{H M}\left(T s_{j}, T s_{i}\right)>0$ iff $(i=1$ and $j>2)$ or $(j>i>1)$ so we have the following two cases.

Case 1 . If $j>2$ and $i=1$, we have

$$
\begin{aligned}
\frac{P_{H M}\left(T s_{j}, T s_{1}\right)\left(1+e^{P_{H M}\left(T s_{j}, T s_{1}\right)}\right)}{d\left(s_{j}, s_{1}\right) e^{d\left(s_{j}, s_{1}\right)}} & \leq \frac{P_{H M}\left(T s_{j}, T s_{1}\right)\left(2 e^{P_{H M}\left(T s_{j}, T s_{1}\right)}\right)}{d\left(s_{j}, s_{1}\right) e^{d\left(s_{j}, s_{1}\right)}} \\
& =\frac{\left(s_{j-1}-s_{1}\right)\left(2 e^{s_{j-1}-s_{1}}\right)}{\left(s_{j}-s_{1}\right)\left(e^{s_{j}-s_{1}}\right)} \\
& =\frac{\left(s_{j-1}-s_{1}\right)\left(2 e^{s_{j-1}-s_{j}}\right)}{\left(s_{j}-s_{1}\right)} \\
& =\frac{\left(j^{2}-j-2\right)\left(2 e^{-j}\right)}{\left(j^{2}-j-2\right)}<2 e^{-j}<2 e^{-1}<1 .
\end{aligned}
$$


That is,

$$
\frac{P_{H M}\left(T s_{j}, T s_{1}\right)\left(1+e^{P_{H M}\left(T s_{j}, T s_{1}\right)}\right)}{d\left(s_{j}, s_{1}\right) e^{d\left(s_{j}, s_{1}\right)}}<1,
$$

which implies that

$P_{H M}\left(T s_{j}, T s_{1}\right) \leq d\left(T s_{j}, T s_{1}\right) e^{d}\left(s_{j}, s_{1}\right)-d\left(T s_{j}, T s_{1}\right) e^{d}\left(T s_{j}, T s_{1}\right)$.

Case 2. If $j>i>1$, then, we have

$$
\begin{aligned}
\frac{P_{H M}\left(T s_{j}, T s_{i}\right)\left(1+e^{P_{H M}\left(T s_{j}, T_{i}\right)}\right)}{d\left(s_{j}, s_{i}\right) e^{d\left(s_{j}, s_{i}\right)}} & \leq \frac{P_{H M}\left(T s_{j}, T s_{i}\right)\left(2 e^{P_{H M}\left(T s_{j}, T s_{i}\right.}\right)}{d\left(s_{j}, s_{i}\right) e^{d\left(s_{j}, s_{i}\right)}} \\
& =\frac{\left(s_{j-1}-s_{i-1}\right)\left(2 e\left(s_{j-1}-s_{i-1}\right)\right)}{\left(s_{j}-s_{i}\right) e\left(s_{j}-s_{i}\right)} \\
& =\frac{\left(s_{j-1}-s_{i-1}\right)\left(2 e\left(s_{j-1}-s_{i-1}-s_{j}+s_{i}\right)\right)}{\left(s_{j}-s_{i}\right)} \\
& =\frac{(j+i-1)\left(2 e^{i-j}\right)}{(j+i+1)} \leq 2 e^{i-j} \leq 2 e^{-1}<1,
\end{aligned}
$$

that is,

$$
\frac{P_{H M}\left(T s_{j}, T s_{i}\right)\left(1+e^{P_{H M}\left(T s_{j}, T s_{i}\right)}\right)}{d\left(s_{j}, s_{i}\right) e^{d\left(s_{j}, s_{i}\right)}} \leq 1,
$$

therefore,

$$
P_{H M}\left(T s_{j}, T s_{i}\right)<d\left(s_{j}, s_{i}\right) e^{d}\left(s_{j}, s_{i}\right)-P_{H M}\left(T s_{j}, T s_{i}\right) e^{P_{H M}\left(T s_{j}, T s_{i}\right)} .
$$

Hence, the condition of Theorem 15 is satisfied. Also, $s_{1}=1$ is a fixed point of $T$.

Example 2. Suppose that $M=[0, \infty), d(s, t)=|s-t|$ for all $s$, $t \in M$. It is obvious that the pair $(M, d)$ is a CMS. Define a multivalued mapping $T$ by

$$
T(s)=\left\{\frac{s}{2}\right\},
$$

then, we have

$$
\begin{gathered}
P_{H M}(T s, T t)=\frac{1}{2}|s-t|=\frac{1}{2} d(s, t), \\
d^{2}(s, t)-P_{H M}^{2}(T s, T t)=d^{2}(s, t)-\frac{1}{4} d^{2}(s, t)=\frac{3}{4} d^{2}(s, t),
\end{gathered}
$$

it follows from (37) and (38) that

$$
P_{H M}(T s, T t) \leq d^{2}(s, t)-P_{H M}^{2}(T s, T t), \quad \forall s, t \in M .
$$

Hence, $T$ satisfies the condition of Theorem 15 with $\varphi($ $p)=p^{2}$.

Remark 16. Theorem 15 upgrades the Nadler fixed point result in a finite-dimensional space. Indeed, if $T$ holds condition of Nadler's theorem, then for $s, t \in M$, we have

$$
P_{H M}(T s, T t) \leq c d(s, t) \leq \frac{c d(s, t)}{1+c-\sqrt{c}},
$$

or, equivalently,

$$
(1-\sqrt{c}) P_{H M}(T s, T t)+c P_{H M}(T s, T t) \leq c d(s, t)
$$

yields

$$
P_{H M}(T s, T t) \leq \frac{c}{1-\sqrt{c}} d(s, t)-\frac{c}{1-\sqrt{c}} P_{H M}(T s, T t) .
$$

Taking $\varphi(p)=c p /(1-\sqrt{ } c)$, we get

$$
P_{H M}(T s, T t) \leq \varphi(d(s, t))-\varphi\left(P_{H M}(T s, T t)\right),
$$

moreover, for each $s \in M, T(s) \in C B(M)$ but $M$ is a finitedimensional space; therefore, $T(s)$ is compact, and hence, by Theorem 15, there is a fixed point of $T$.

Let $\Omega$ be the set of all mappings $f: R \longrightarrow(0 \infty)$ having the same stipulations (1)-(3) of Theorem 6 . Note: by property (2), of Theorem 6 , we have $f(a)=1$ if and only if $a=$ 0 .

Example 3. All the functions in the following belong to $\Omega$.

(i) $f_{1}(s)=1+\tanh s$

(ii) $f_{2}(s)=e^{s}$

(iii) $f_{3}(s)= \begin{cases}1+\ln (1+s), & \text { if } s \in 0, \infty) \\ e^{s}, & \text { if } s \in(-\infty, 0]\end{cases}$

The lemmas in the following help us to supplement the theoretical results.

Lemma 17. Assume that $(M, d)$ is a CMS and $T: M \longrightarrow N$ $(M)$ is a multivalued mapping. Let $\phi: M \longrightarrow R$ be a function defined in Theorem 6 and $f \in \Omega$. Define the relation " $\leq$ " on $M$ so that

$$
s \leq t \Leftrightarrow f((d(s, t))) \leq \frac{f(\phi(s))}{f(\phi(t))},
$$

then, the relation " $\leq$ " is a partial order on $M$ and $M$ is a partial order space. 
Proof. Since $f(a)=1$ iff $a=0$, then, we have

$$
f(d(s, s))=f(0)=1=\frac{f(\phi(s))}{f(\phi(s))},
$$

this shows that $s \leq s$.

If $s \leq t$ and $t \leq s$, then

$$
\begin{aligned}
& f(d(s, t)) \leq \frac{f(\phi(s))}{f(\phi(t))}, \\
& f(d(t, s)) \leq \frac{f(\phi(t))}{f(\phi(s))},
\end{aligned}
$$

because $d(t, s)=d(s, t)$; thus, $f(d(t, s))=1$. Moreover, $f(a)$ $=1$ iff $a=0$; therefore, $d(t, s)=0$; this implies that $s=t$.

Finally, if $s \leq t$ and $t \leq u$, then

$$
\begin{gathered}
f(d(s, t)) \leq \frac{f(\phi(s))}{f(\phi(t))}, \\
\text { and } f(d(t, u)) \leq \frac{f(\phi(t))}{f(\phi(u))},
\end{gathered}
$$

it follows from $f(a+b) \leq f(a) \cdot f(b)$ that

$f(d(s, u)) \leq f(d(s, t)+d(t, u)) \leq f(d(s, t) \cdot f(d(t, u))) \leq \frac{f(\phi(s))}{f(\phi(u))}$

From the above results, we conclude that " $\leq$ " is a partial order on $M$.

Lemma 18. Let the pair $(M, d)$ be a CMS and $T: M \longrightarrow N$ $(M)$ be a multivalued mapping. Consider $\phi: M \longrightarrow R$ be a function defined in Theorem $6, f \in \Omega$ and $\eta:[0, \infty) \longrightarrow 0$, $\infty)$ is a nondecreasing, continuous, and subadditive function that ensures that $\eta^{-1}(\{0\})=\{0\}$. Define the relation " $\leq$ " on $M$ by

$$
s \leq t \Leftrightarrow f(\eta(d(s, t))) \leq \frac{f(\phi(s))}{f(\phi(t))},
$$

then, the relation " $\leq$ " is a partial on $M$ and $M$ is a partial order space.

Proof. Since $f(a)=1$ iff $a=0$ and $\eta^{-1}(\{0\})=\{0\}$, then, we get

$$
f(\eta(d(s, s)))=f(\eta(0))=f(0)=1=\frac{f(\phi(s))}{f(\phi(s))},
$$

this implies that $s \leq s$.
If $s \leq t$ and $t \leq s$, then

$$
\begin{aligned}
& f(\eta(d(s, t))) \leq \frac{f(\phi(s))}{f(\phi(t))}, \\
& f(\eta(d(t, s))) \leq \frac{f(\phi(t))}{f(\phi(s))} .
\end{aligned}
$$

Since $d(t, s)=d(s, t)$, thus $f(d(t, s))=1$. Moreover, $f(a)$ $=1$ iff $a=0$ and $\eta^{-1}(\{0\})=\{0\}$; therefore, $d(t, s)=0$, i.e., $s$ $=t$.

Again, if $s \leq t$ and $t \leq u$, then

$$
\begin{gathered}
f(\eta(d(s, t))) \leq \frac{f(\phi(s))}{f(\phi(t))}, \\
f(\eta(d(t, u))) \leq \frac{f(\phi(t))}{f(\phi(u))},
\end{gathered}
$$

it follows from $f(a+b) \leq f(a) \cdot f(b)$, and $\eta$ is nondecreasing and subadditive that

$$
\begin{aligned}
f(\eta(d(s, u))) & \leq f(\eta(d(s, t)+d(t, u))) \\
& \leq f(\eta(d(s, t))+\eta(d(t, u))) \\
& \leq f(\eta(d(s, t))) \cdot f(\eta(d(t, u))) \leq \frac{f(\phi(s))}{f(\phi(u))},
\end{aligned}
$$

and this completes the required. So, " $\leq$ " is a partial order on $M$.

Now, we can state and prove the next main theorems.

Theorem 19. Let $(M, d)$ be a CMS, $T: M \longrightarrow N(M)$ be a multivalued mapping, $\phi: M \longrightarrow R$ be a function defined as Theorem 6, and $f \in \Omega$. If for all $s \in M$, there is $t \in T(s)$ so that

$$
f(d(s, t)) \leq \frac{f(\phi(s))}{f(\phi(t))}
$$

then, the mapping $T$ has a fixed point in $M$.

Proof. Since $M$ is a partial order space, then we need to prove only that $M$ has a maximal element. Suppose that $\left\{s_{\alpha}\right\}_{\alpha \in I}$ is increasing sequence in $M$, that is, for $\alpha, \beta \in I$ with $\alpha \leq \beta$ then $s_{\alpha} \leq s_{\beta}$ from (54), we have $\left\{\phi\left(s_{\alpha}\right)\right\}_{\alpha \in I}$ is decreasing, since $\phi$ is bounded as follows then $\inf _{\alpha \in I} \phi\left(s_{\alpha}\right)$ holds. Assume that $\alpha_{n}$ is increasing sequence in $I$ that ensures

$$
\lim _{n \longrightarrow \infty} \phi\left(s_{\alpha_{n}}\right)=\inf _{\alpha \in I} \phi\left(s_{\alpha}\right)=r .
$$

Now, for $m, n \in \mathbb{N}$ with $m \geq n$, then $\alpha_{n} \leq \alpha_{m}$ and $\left\{s_{\alpha_{n}}\right\}$ $\leq\left\{s_{\alpha_{m}}\right\}$. 
Applying (54), we get

$$
\begin{aligned}
f\left(d\left(s_{\alpha_{n}}, s_{\alpha_{m}}\right)\right) & =f\left(\sum_{i=n}^{m-1} d\left(s_{i}, s_{i+1}\right)\right) \\
& \leq \Pi_{i=n}^{m-1} f\left(d\left(s_{i}, s_{i+1}\right)\right) \\
& \leq \Pi_{i=n}^{m-1} \frac{f\left(\phi\left(s_{i}\right)\right)}{f\left(\phi\left(s_{i+1}\right)\right)}=\frac{f\left(\phi\left(s_{n}\right)\right)}{f\left(\phi\left(s_{m}\right)\right)} .
\end{aligned}
$$

As $m, n \longrightarrow \infty$ in the above inequality, we have $\lim _{n, m \longrightarrow \infty} f d\left(s_{\alpha_{n}}, s_{\alpha_{m}}\right)=1$. Since $f$ is continuous so, we obtain

$$
\lim _{n, m \longrightarrow \infty} d\left(s_{\alpha_{n}}, s_{\alpha_{m}}\right)=0
$$

Hence, $\left\{s_{\alpha_{n}}\right\}$ is a Cauchy sequence in $M$. Since $M$ is complete then there exists $s \in M$ so that

$\left\{s_{\alpha_{n}}\right\} \longrightarrow s$ as $n \longrightarrow \infty$. Thus, $s$ is an upper bound for $\left\{s_{\alpha_{n}}\right\}$ for each $n \geq 1$. Now, we want to show that $s$ is also an upper bound for $\left\{s_{\alpha}\right\}$. Suppose that $\beta \in I$ with $s_{\alpha_{n}} \leq s_{\beta}$ for each $n \geq 1$. Then, by (54), we obtain that $\phi\left(s_{\beta}\right) \leq \phi\left(s_{\alpha_{n}}\right)$ for each $n \geq 1$. Also, from (54), we have

$$
\phi\left(s_{\beta}\right)=\inf _{\alpha \in I} \phi\left(s_{\alpha}\right)=r
$$

Since

$$
f\left(d\left(s_{\alpha_{n}}, s_{\beta}\right)\right) \leq \frac{f\left(\phi\left(s_{\alpha_{n}}\right)\right)}{f\left(\phi\left(s_{\beta}\right)\right)}
$$

then, by taking the limit as $n \longrightarrow \infty$, we get $\lim _{n \longrightarrow \infty} s_{\alpha_{n}}=s_{\beta}$. Hence, for each $\alpha \in I$, there is $n \geq 1$ so that $s_{\alpha} \leq s_{\alpha_{n}}$, also $s_{\alpha_{n}}$ $\leq s$, yields $s_{\alpha} \leq s$ for each $\alpha \in I$. Thus, $s$ is an upper bound for $\left\{s_{\alpha}\right\}_{\alpha \in I}$. By Zorn's lemma, $M$ has a maximal element $s_{0}$. The condition $t_{\mathrm{o}} \in T\left(s_{\mathrm{o}}\right)$ implies that

$$
f\left(d\left(s_{\circ}, t_{\circ}\right)\right) \leq \frac{f\left(\phi\left(s_{\circ}\right)\right)}{f\left(\phi\left(t_{\circ}\right)\right)}
$$

this shows that $s_{\mathrm{o}} \leq t_{\mathrm{o}}$. Since $s_{\mathrm{o}}$ is maximal, therefore, $s_{\mathrm{o}}=t_{\mathrm{o}}$. Therefore, $s_{\circ} \in T\left(s_{\circ}\right)$. This ends the proof.

Theorem 20. Let $(M, d)$ be a CMS, $T: M \longrightarrow N(M)$ be a multivalued mapping, $\phi: M \longrightarrow R$ be a function defined as Theorem $6, f \in \Omega$, and $\eta:[0, \infty) \longrightarrow 0, \infty)$ be a nondecreasing continuous subadditive function such that $\eta^{-1}(\{0\})=\{0\}$ . If for any $s \in M$, there is $t \in T(s)$ so that

$$
f(\eta(d(s, t))) \leq \frac{f(\phi(s))}{f(\phi(t))}
$$

then, there is a fixed point of $T$ in $M$.

Proof. By the same manner of the proof of Theorem 19, we can easily show that there is a maximal point $s_{\text {o of partial }}$ order space $M$, and by hypothesis, there is $t_{0} \in T\left(s_{\circ}\right)$ that ensures

$$
f\left(\eta\left(d\left(s_{\circ}, t_{\mathrm{o}}\right)\right)\right) \leq \frac{f\left(\phi\left(s_{\mathrm{o}}\right)\right)}{f\left(\phi\left(t_{\mathrm{o}}\right)\right)}
$$

This implies that $s_{\circ} \leq t_{\circ}$. As $s_{\circ}$ is a maximal element of $M$, therefore, $s_{\circ}=t_{\mathrm{o}}$; hence, $s_{\mathrm{o}} \in T\left(s_{\mathrm{o}}\right)$. This completes the proof.

\section{Supportive Application}

Here, we use Theorem 15 with $\varphi(p)=p^{2}$ to determine the existence of a solution to the Volterra-type integral inclusion of the following form:

$$
s(q) \in \int_{a}^{q} W(q, j, s(j)) d j+g(q), q \in[a, b]
$$

where $W:[a, b] \times[a, b] \times \mathbb{R} \longrightarrow P_{c v}(\mathbb{R}), P_{c v}(\mathbb{R})$ refers to the class of nonempty compact and convex subset of $\mathbb{R}$ and $g$ $:[a, b] \longrightarrow \mathbb{R}$ is a continuous function.

Let $M=C([a, b], \mathbb{R})$ be the space of all continuous realvalued functions on $[a, b]$. Define the distance

$$
d(s, t)=\sup _{q \in[a, b]}|s(q)-t(q)|
$$

for all $s \in M$. It is clear that the pair $(M, d)$ is a metric space.

We shall consider Problem (63) under the hypotheses as follows:

(1) $W:[a, b] \times[a, b] \times \mathbb{R} \longrightarrow P_{c v}(\mathbb{R})$ and $g:[a, b] \longrightarrow$ $\mathbb{R}$ are continuous functions, as well as $W$ is compact and convex

(2) There is a continuous function $l: M \longrightarrow 0 \infty)$ so that

$$
P_{H M}(W(q, j, s(j)), W(q, j, t(j))) \leq l(j)\left(\frac{-1+\sqrt{1+4(s(j)-t(j))^{2}}}{2}\right)
$$

for each $q, j \in[a, b]$ and $s, t \in M$

(3) We have $\sup _{t \in[a, b]} \int_{a}^{t} l(j) d j \leq 1$

Now, our main theorem in this part becomes valid for showing.

Theorem 21. Under the assumptions (1)-(3), Problem (63) has a solution on $M$. 
Proof. Define the multivalued mapping $T: C([a, b], R) \longrightarrow$ $C P(C([a, b], R))$ by

$$
\operatorname{Ts}(q)=\left\{u \in M: u \in \int_{a}^{q} W(q, j, s(j)) d j+g(q), q \in[a, b]\right\} .
$$

The unique solution of Problem (63) is equivalent to find a fixed point of $T$ in $M$.

It is obvious that $T s$ is compact because for any $s \in M$, $W(., ., s)$ is lower semicontinuous. For clarification, let $W_{s}$ $=W(q, j, s(j))$, for each $q, j \in[a, b]$. Thus, $W_{s}:[a, b] \times[a, b]$ $\longrightarrow P_{c v}(\mathbb{R})$, by Michael selection theorem [38], there is a continuous function $m_{s}:[a, b] \times[a, b] \longrightarrow \mathbb{R}$ so that $m_{s}(q, j$ )$\in W_{s}(q, j)$. It follows that $\int_{a}^{q} m_{s}(q, j) d j+g(q) \in T s(q)$; this implies that $T s$ is nonempty. Hence, it is compact.

Proof. Suppose that $s, u \in M$ such that $u \in T s$. Then, for each $q, j \in[a, b], m_{s}(q, j) \in W_{s}(q, j)$ and

$$
u(q)=\int_{a}^{q} m_{s}(q, j) d j+g(q), \quad \forall q \in[a, b]
$$

Now, by condition (1), there exist $v_{t}(q, j) \in W_{t}(q, j)$ such that

$\left|m_{s}(q, j)-v_{t}(q, j)\right| \leq l(j) \frac{-1+\sqrt{1+4|s(j)-t(r)|^{2}}}{2}, \quad \forall q, j \in[a, b]$.

Let us define the multivalued operator $O$ by

$$
\begin{aligned}
O(q, j) & =W_{t}(q, j) \cap\left\{w \in \mathbb{R}:\left|m_{s}(q, s)-w\right|\right. \\
& \left.\leq q(j) \frac{-1+\sqrt{1+4|s(j)-t(j)|^{2}}}{2}\right\}, \quad \forall q, j \in[a, b] .
\end{aligned}
$$

Since $T$ is lower semicontinuous, then there exist $m_{t}:$ [ $a, b] \times[a, b] \longrightarrow R$ such that for all $q, j \in[a, b]$, $m_{t}(q, j) \in W_{t}(q, j)$. Thus, for any $r \in M$,

$r(q)=\int_{a}^{q} m_{t}(q, j) d j+g(q) \in \int_{a}^{q} W(q, j, t(j)) d j+g(q), q \in[a, b]$.
By condition (3), we can write

$$
\begin{aligned}
|u(q)-r(q)| & \leq \int_{a}^{q}\left|m_{s}(q, j)-m_{t}(q, j)\right| d j \\
& \leq \int_{a}^{q} l(j) \frac{-1+\sqrt{1+4|s(j)-t(j)|^{2}}}{2} d j \\
& \leq \int_{a}^{q} l(j) \frac{-1+\sqrt{1+4)\|s(j)-t(j)\|^{2}}}{2} d j \\
& =\frac{-1+\sqrt{1+4\|s(j)-t(j)\|^{2}}}{2} \int_{a}^{q} l(j) d j \\
& \leq\left(\frac{-1+\sqrt{1+4\|s(j)-t(j)\|^{2}}}{2}\right)\left(\sup \int_{a}^{q} l(j) d j\right) \\
& \leq \frac{-1+\sqrt{1+4\|s(j)-t(j)\|^{2}}}{2} .
\end{aligned}
$$

This implies that

$$
d(u(q), r(q)) \leq \frac{-1+\sqrt{1+4[d(s(j), t(j))]^{2}}}{2}
$$

Now, by exchanging the rule of $s$ and $t$, we have

$$
P_{H M}(T s, T t) \leq \frac{-1+\sqrt{1+4[d(s(j), t(j))]^{2}}}{2}, \quad \forall s, t \in M,
$$

yielding

$$
\left(1+2 P_{H M}(T s, T t)\right)^{2} \leq 1+4[d(s, t)]^{2}
$$

is equivalent to

$$
P_{H M}(T s, T t)+\left[P_{H M}(T s, T t)\right]^{2} \leq[d(s, t)]^{2} .
$$

Therefore,

$$
P_{H M}(T s, T t) \leq[d(s, t)]^{2}-\left[P_{H M}(T s, T t)\right]^{2}, \quad \forall s, t \in M .
$$

Therefore, all the conditions of Theorem 15 are fulfilled. Therefore, the operator $T$ has a fixed point which is a solution to Problem (63).

\section{Data Availability}

No data were used. 


\section{Conflicts of Interest}

The authors declare that they have no competing interests concerning the publication of this article.

\section{Authors' Contributions}

All authors contributed equally and significantly in writing this article.

\section{Acknowledgments}

The fourth author Habes Aslamir extends his appreciation to the College of Business Administration-Finance Department, Dar Al Uloom University for funding this work.

\section{References}

[1] E. I. Fredholm, "Sur une classe d'équations fonctionnelles," Acta mathematica, vol. 27, pp. 365-390, 1903.

[2] R. Sevinik-Adıguzel, E. Karapınar, and İ. M. Erhan, "A solution to nonlinear Volterra integro-dynamic equations via fixed point theory," Filomat, vol. 33, no. 16, pp. 5331-5343, 2019.

[3] R. Sevinik Adigüzel, Ü. Aksoy, E. Karapinar, and İ. M. Erhan, "On the solution of a boundary value problem associated with a fractional differential equation," Mathematical Methods in the Applied Sciences, vol. 2020, article mma.6652, 12 pages, 2020.

[4] R. Sevinik-Adıgüzel, Ü. Aksoy, E. Karapınar, and İ. M. Erhan, "Uniqueness of solution for higher-order nonlinear fractional differential equations with multi-point and integral boundary conditions," Revista de la Real Academia de Ciencias Exactas, Físicas y Naturales. Serie A. Matemáticas, vol. 115, no. 3, article 155, p. 16, 2021.

[5] M. D. Rus, "A note on the existence of positive solution of Fredholm integral equations," Fixed Point Theory, vol. 5, pp. 369-377, 2004.

[6] H. Aydi, M. Jleli, and B. Samet, "On positive solutions for a fractional thermostat model with a convex-concave source term via \$\$psi \$\$-Caputo fractional derivative," Mediterranean Journal of Mathematics, vol. 17, no. 1, p. 16, 2020.

[7] E. Karapinar, P. Kumari, and D. Lateef, "A new approach to the solution of the Fredholm integral equation via a fixed point on extended b-metric spaces," Symmetry, vol. 10, no. 10, p. 512,2018

[8] H. A. Hammad and M. De la Sen, "A solution of Fredholm integral equation by using the cyclic $\eta_{\mathrm{s}}^{\mathrm{q}}$ - rational contractive mappings technique in $\mathrm{b}$-metric-like spaces," Symmetry, vol. 11, no. 9, pp. 1184-1222, 2019.

[9] H. A. Hammad and M. De la Sen, "Solution of nonlinear integral equation via fixed point of cyclic $\alpha_{\mathrm{s}}^{\mathrm{q}}$ - rational contraction mappings in metric-like spaces," Bulletin of the Brazilian Mathematical Society, New Series, vol. 51, no. 1, pp. 81-105, 2020.

[10] S. K. Panda, E. Karapinar, and A. Atangana, "A numerical schemes and comparisons for fixed point results with applications to the solutions of Volterra integral equations in dislocatedextendedb- metricspace," Alexandria Engineering Journal, vol. 59, no. 2, pp. 815-827, 2020.

[11] S. K. Panda, T. Abdeljawad, and K. Kumara Swamy, "New numerical scheme for solving integral equations via fixed point method using distinct ( $\omega$-F)-contractions," Alexandria Engineering Journal, vol. 59, no. 4, pp. 2015-2026, 2020.

[12] M. I. Berenguer, M. V. F. Munoz, A. I. G. Guillem, and M. R. Galan, "Numerical treatment of fixed point applied to the nonlinear Fredholm integral equation," Fixed Point Theory and Applications, vol. 2009, 638 pages, 2009.

[13] S. B. Nadler Jr., "Multi-valued contraction mappings," Pacific Journal of Mathematics, vol. 30, no. 2, pp. 475-488, 1969.

[14] A. Hussain, M. Ishfaq, and T. Kanwal, "Gerghaty type results via simulation and $\mathscr{C}$-class function with application," The International Journal of Nonlinear Analysis and Applications (IJNAA), vol. 12, pp. 1057-1071, 2021.

[15] A. Branciari, "A fixed point theorem for mappings satisfying a general contractive condition of integral type," International Journal of Mathematics and Mathematical Sciences, vol. 29, no. 9, Article ID 641824, 536 pages, 2002.

[16] R. P. Agarwal, D. O. O'regan, and N. Shahzad, "Fixed point theory for generalized contractive maps of Meir-Keeler type," Mathematische Nachrichten, vol. 276, no. 1, pp. 3-22, 2004.

[17] S. Chauhan, M. Imdad, C. Vetro, and W. Sintunavarat, "Hybrid coincidence and common fixed point theorems in Menger probabilistic metric spaces under a strict contractive condition with an application," Applied Mathematics and Computation, vol. 239, pp. 422-433, 2014.

[18] M. A. Kutbi and W. Sintunavarat, "The existence of fixed point theorems via $\omega$-distance and -admissible mappings and applications," Abstract and Applied Analysis, vol. 2013, Article ID 165434, 8 pages, 2013.

[19] M. U. Ali and T. Kamran, "On $\left(\alpha^{*}, \psi\right)$-contractive multivalued mappings," Fixed Point Theory and Applications, vol. 2013, no. 1, Article ID 472, 2013.

[20] J. P. Aubin and J. Siegel, "Fixed points and stationary points of dissipative multivalued maps," Proceedings of the American Mathematical Society, vol. 78, no. 3, pp. 391-398, 1980.

[21] H. Covitz and S. B. Nadler Jr., "Multi-valued contraction mappings in generalized metric spaces," Israel Journal of Mathematics, vol. 8, no. 1, pp. 5-11, 1970.

[22] L. V. Hot, "Fixed point theorems for multi-valued mapping," Commentationes Mathematicae Universitatis Carolinae, vol. 23, pp. 137-145, 1982.

[23] M. U. Ali, T. Kamran, and E. Karapınar, "Fixed point of $\alpha-\psi$ contractive type mappings in uniform spaces," Fixed Point Theory and Applications, vol. 2014, no. 1, Article ID 150, 2014.

[24] W. S. du and E. Karapinar, "A note on Caristi-type cyclic maps: related results and applications," Fixed Point Theory and Applications, vol. 2013, no. 1, Article ID 643, 2013.

[25] A. Petrusel and A. Sîntamarian, "Single-valued and multivalued Caristi type operators," PUBLICATIONES MATHEMATICAE-DEBRECEN, vol. 60, pp. 167-177, 2002.

[26] A. Hussain, S. Yaqoob, T. Abdeljawad, and H. Ur Rehman, "Multivalued weakly Picard operators via simulation functions with application to functional equations," AIMS Mathematics, vol. 6, no. 3, pp. 2078-2093, 2021.

[27] E. Karapinar, "Generalizations of Caristi Kirk's theorem on partial metric spaces," Fixed Point Theory and Applications, vol. 2011, no. 1, Article ID 4, 7 pages, 2011.

[28] T. Abdeljawad and E. Karapinar, "Quasicone metric spaces and generalizations of Caristi Kirk's theorem," Fixed Point Theory and Applications, vol. 2009, no. 1, Article ID 574387, 2009. 
[29] J. R. Jachymski, "Converses to fixed point theorems of Zermelo and Caristi," Nonlinear Analysis, vol. 52, no. 5, pp. 1455-1463, 2003.

[30] F. Khojasteh, E. Karapinar, and H. Khandani, "Some applications of Caristi's fixed point theorem in metric spaces," Fixed Point Theory and Applications, vol. 2016, no. 1, Article ID 16, 2016.

[31] E. Karapınar, F. Khojasteh, and Z. Mitrović, “A proposal for revisiting Banach and Caristi type theorems in b-metric spaces," Mathematics, vol. 7, no. 4, pp. 308-314, 2019.

[32] M. U. ALI, T. Kamran, W. Sintunavarat, and P. Katchang, "Mizoguchi-Takahashi's fixed point theorem with $\alpha, \eta$ function," Abstract and Applied Analysis, vol. 2013, Article ID 418798, 4 pages, 2013.

[33] J. Caristi, "Fixed point theorems for mappings satisfying inwardness conditions," Transactions of the American Mathematical Society, vol. 215, pp. 241-251, 1976.

[34] Y. Feng and S. Liu, "Fixed point theorems for multi-valued contractive mappings and multi-valued Caristi type mappings," Journal of Mathematical Analysis and Applications, vol. 317, no. 1, pp. 103-112, 2006.

[35] H. Isik, B. Mohammadi, M. R. Haddadi, and V. Parvaneh, "On a new generalization of Banach contraction principle with application," Mathematics, vol. 7, no. 9, p. 862, 2019.

[36] H. Kadakal, "Hermite-Hadamard type inequalities for subadditive functions," AIMS Mathematics, vol. 5, no. 2, pp. 930939, 2020.

[37] F. Mirdamad, M. Asadi, and S. Abbasi, "Approximate best proximity for set valued contraction in metric space," Journal of Mathematical Analysis, vol. 9, pp. 53-60, 2018.

[38] E. Michael, "Continuous selections. I," Annals of mathematics, vol. 63, no. 2, pp. 361-382, 1956. 